\title{
Competitions in a Networked Society: Crowdsourcing Collective Design Intelligence
}

\author{
IMDAT AS \\ University of Hartford
}

\begin{abstract}
This paper discusses the reincarnation of the traditional competition model in online crowdsourcing platforms, thanks to an ever-more networked global marketplace. After giving a brief background on competitions, the paper will elaborate on crowdsourcing, discuss the dichotomy between competition and collaboration, and debate the potential impact of artificial intelligence on future competitions. The opportunity to harness collective design intelligence through collaborative features and modifications in the competition protocol suggests a new mode of competitive collaboration. The paper ends with a speculation on how such metamorphosis of traditional competitions can affect the role of the architect, and the future of the architectural practice.
\end{abstract}

\section{INTRODUCTION}

Architecture relies on the logic of abductive reasoning. ${ }^{1}$ The solution space in design is not as clear cut as it is in engineering, for example. Designers are not striving towards a single correct answer, but instead, and quite to the contrary, try to resolve the problem through a unique, original and idiosyncratic solution. Back in my architectural education, a professor in design studio explained the fact that they do not give A grades is because there is always the possibility of a better design solution. Design can be infinitely iterated. Therefore, it is not surprising that competitions have been used throughout history to generate multiple design options to compare, contrast, and choose from.

Nevertheless, people get surprised by the fact that architects even submit designs to competitions, since the chance to win and convert an idea into brick and mortar through competitions is often lower than one percent. Louis Kahn suggested that competitions are therefore a free offering by architects to the larger community, since the majority of projects never get built and architects not paid. ${ }^{2}$ For most architects, design is undeniably a passion, which can be explained by the concept of urges or drives in motivational psychology. Adolf Loos claimed that any creative act serves the sublimation of the creator's urges, and therefore performs functions beyond its apparent value proposition. ${ }^{3}$ Loos's argument, which was originally made against ornament in modernist architecture, may also explain why designers participate in design challenges, as competitions offer fierce battle grounds for "creative acts" to outperform.

Many architects made breakthroughs in their careers and established their very existence through competitions. Gunther Behnisch, who won the prestigious competition for the Munich Olympic Park in 1968, for example, participated in more than 800 competitions ever since. Farshid Moussavi, a renowned contemporary practitioner, participated in more than 200 competitions over her career, and values competitions for generating "creative leaps." However, there are also well-known architects who avoided competitions altogether, like Frank Lloyd Wright, Louis Kahn, or William Robert Ware, who wrote back in 1899:

Every competition ... costs the profession hundreds of thousands of dollars, most of which falls upon men who ill can afford the loss. It is cruel and heartbreaking, when fifty or a hundred sets of drawings are submitted for judgement, to consider that... all but one... have labored in vain, and that out of all the schemes only half a dozen can possibly receive any serious consideration... Thus the profession grows and travails night and day, year in and year out, under the strain of sacrifices it can ill afford to make. No wonder that the system of competitions has come to be regarded as a sort of nightmare, as an incubus or vampire, stifling the breath of professional life, and draining its blood. ${ }^{4}$

-William Robert Ware

Despite such critique, competitions are increasingly more accepted within the larger community of project-owners, and gradually turn into the norm as a modus operandi for the production of important edifices around the globe. A new embodiment of competitions -in the form of online crowdsourcing platforms- is addressing the demand of smaller-scale design challenges and opening up the fair competition protocol to everyday design challenges.

\section{CROWDSOURCING ARCHITECTURE}

Architectural practice is constantly going through a transition of technologies, e.g. novel graphic communication tools, new generative design and practice models, etc. - new methods, techniques and ideas are tested out continuously. This endless flux of changes coupled with the design talent available throughout the world makes crowdsourcing an attractive vehicle for design acquisition. In general, crowdsourcing makes use of a swarm of qualified professionals around the globe to resolve a particular problem. This can range from aggregating and editing Wikipedia entries, to solving wicked science problems (innocentive.com), or generating new logos (99designs.com), and many more. Crowds are solicited to "develop a new product or service, 

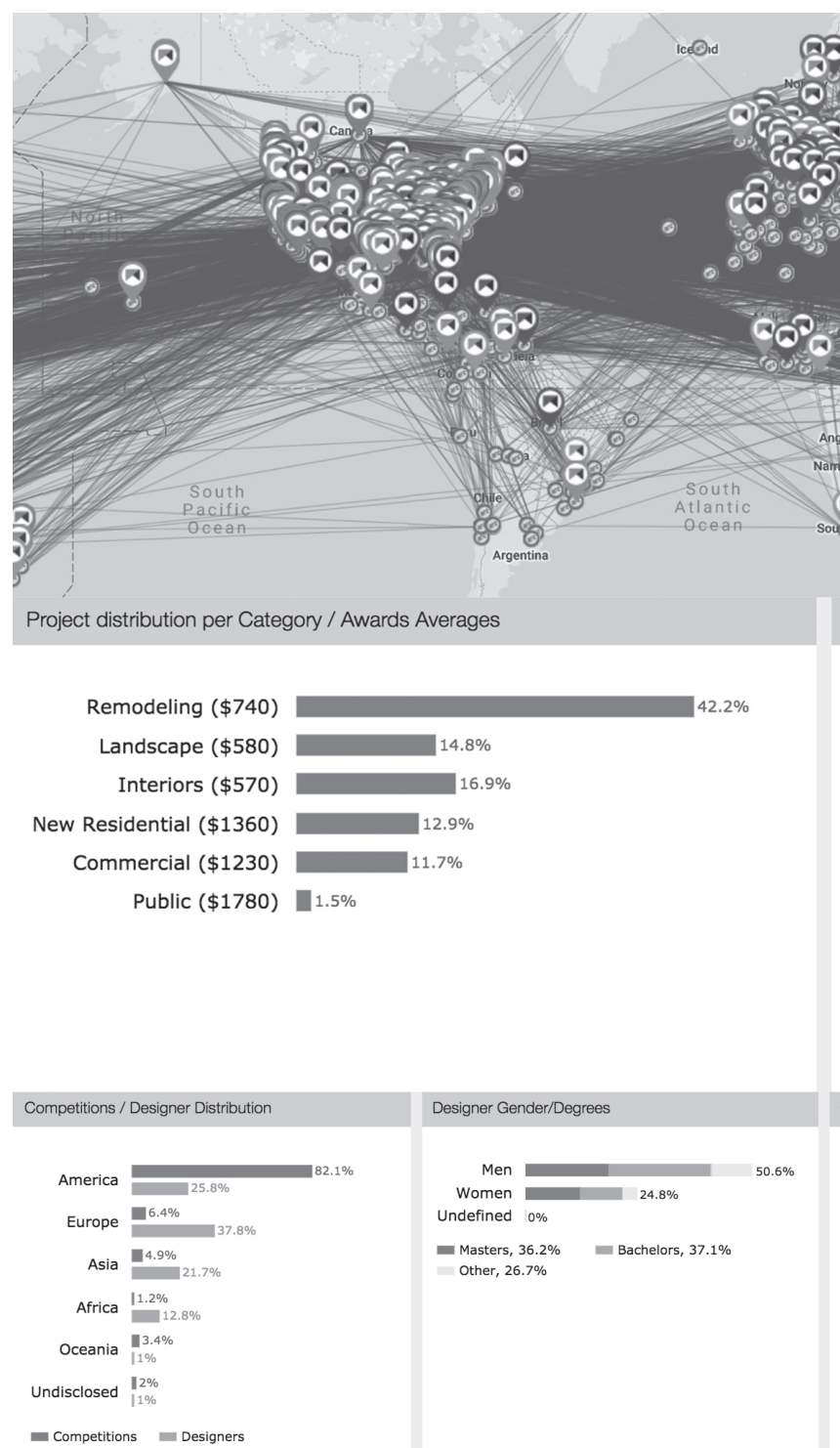

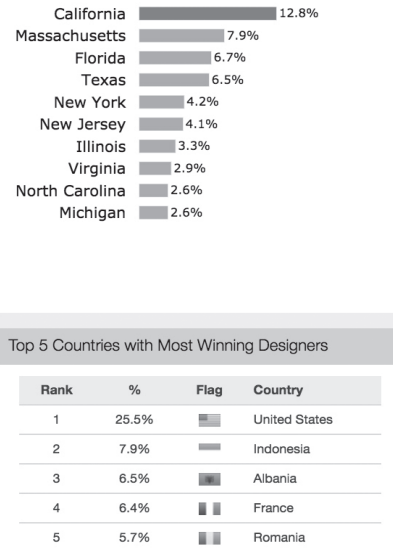

\section{Designer Gender/Degrees}

$$
\text { Me }
$$$$
\text { Women }
$$$$
\text { Undefined } 0 \%
$$$$
\text { - Masters, } 36.2 \%
$$$$
\text { Other, } 26.7 \%
$$

Bachelors, 37.1\%

Other, $26.7 \%$

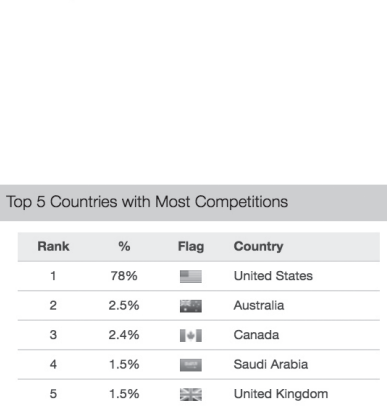

Top 5 Competitions with most Entries

$$
\begin{array}{ccll}
\hline \text { Rank } & \text { Entries } & \text { Title } & \\
\hline 1 & 48 & \begin{array}{l}
\text { Design Modern House in } \\
\text { Madrid Hills (ENG-ESP- } \\
\text { RUS) }
\end{array} & \begin{array}{l}
\text { see } \\
\text { projects }
\end{array} \\
\hline 2 & 45 & \text { Floor Plan Layout } & \begin{array}{l}
\text { see } \\
\text { projects }
\end{array} \\
\hline 3 & 44 & \text { Fireplace Wall } & \begin{array}{l}
\text { see } \\
\text { projects }
\end{array} \\
\hline 4 & 42 & \text { MASTER BATH } & \begin{array}{l}
\text { see } \\
\text { projects }
\end{array} \\
\hline 5 & 41 & \text { Bedroom Interlor design } & \begin{array}{l}
\text { see } \\
\text { projects }
\end{array} \\
\text { See Top Competitions > } &
\end{array}
$$

\section{Top 5 Competitions with most Votes}

$$
\begin{array}{ccll}
\hline \text { Rank } & \text { Votes } & \text { Title } & \\
\hline 1 & 64,060 & \begin{array}{l}
\text { Obama Presidential } \\
\text { Center }
\end{array} & \begin{array}{l}
\text { see } \\
\text { projects }
\end{array} \\
\hline 2 & 3,800 & \begin{array}{l}
\text { Curb Appeal for Forest } \\
\text { Vacation Retreat }
\end{array} & \begin{array}{l}
\text { see } \\
\text { projects }
\end{array} \\
\hline 3 & 3,180 & \begin{array}{l}
\text { Off Grid Retirement Home } \\
\text { in Eastern Ontario }
\end{array} & \begin{array}{l}
\text { see } \\
\text { projects }
\end{array} \\
\hline 4 & 3,090 & \begin{array}{l}
\text { New Build - Soft Modern } \\
\text { Duplex }
\end{array} & \begin{array}{l}
\text { see } \\
\text { projects }
\end{array} \\
\hline 5 & 2,060 & \begin{array}{l}
\text { Simple Ultra Modern } \\
\text { Home Dallas, TX }
\end{array} & \begin{array}{l}
\text { see } \\
\text { projects }
\end{array} \\
\hline
\end{array}
$$

Awards go to Designers in:
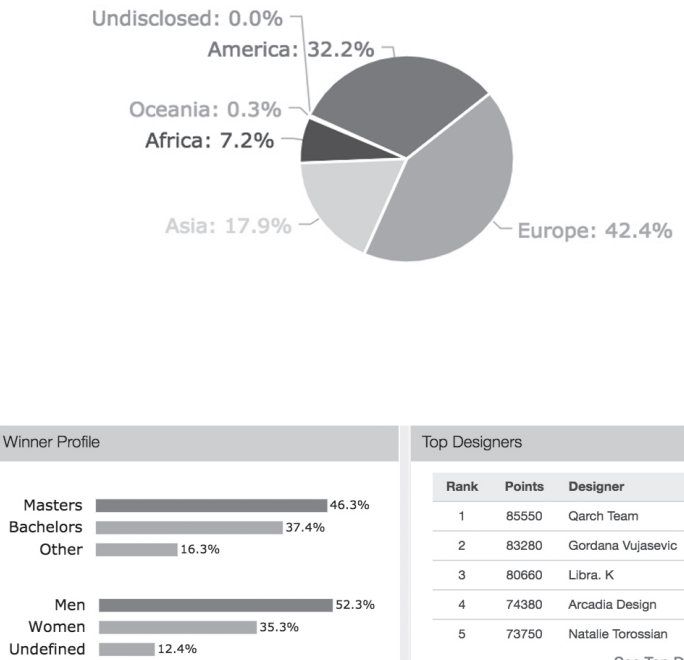

Undefined $12,4 \%$

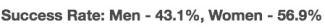

Top 5 Countries with Most Designers

\begin{tabular}{cccl}
\hline Rank & $\%$ & Flag & Country \\
\hline 1 & $22.2 \%$ & $\underline{\underline{E}}$ & United States \\
\hline 2 & $5.9 \%$ & $=$ & India \\
\hline 3 & $5.4 \%$ & $=$ & Egypt \\
\hline 4 & $3.6 \%$ & $\mathbf{C}$ & Turkey \\
\hline 5 & $3.1 \%$ & IU山 & Canada \\
\hline
\end{tabular}

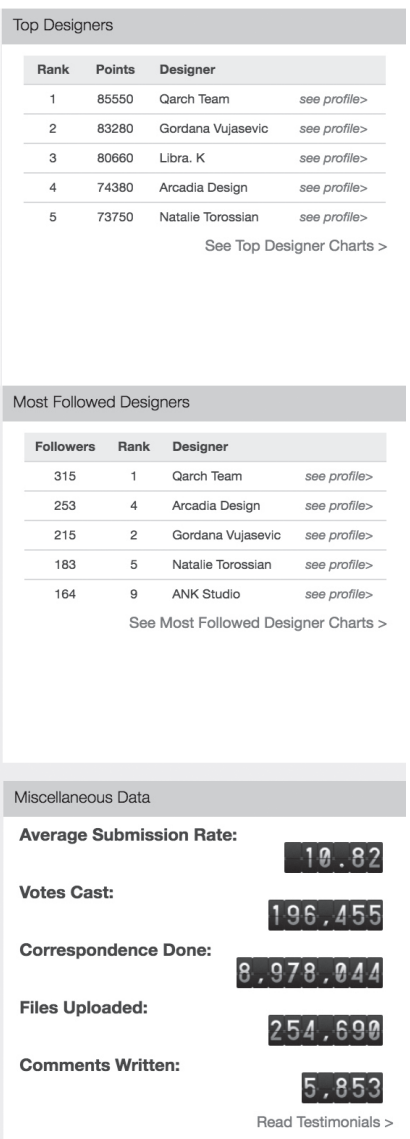

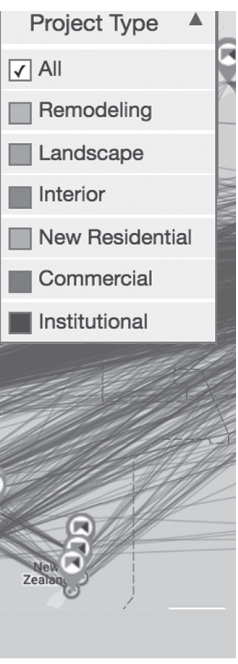

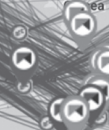


refine a project, calculate or obtain different algorithms, or assist in providing, organizing, or evaluating significant amounts of information in viable data." ${ }^{\prime \prime}$ In architecture, crowdsourcing opens up new opportunities to generate design and facilitate collaboration among designers.

As part of this research we developed Arcbazar.com (Arcbazar), an online crowdsourcing platform, that enables project-owners to launch competitions among designers to work on various design challenges. Our goal was to develop a mechanism that could engage any scale of project in a meaningful and critical way. Project-owners are able to onboard their projects with a few clicks, provide a short description, upload images and dimensions, and set deadlines and monetary awards. During the competition process they can interact with designers via an anonymous communication interface. As of September 2018, Arcbazar gathered more than 20,000 projects worldwide with more than 200,000 renderings, drawings, videos, and millions of communication strings. The types of competitions on the platform were distributed as follows: $42.2 \%$ remodeling, $16.9 \%$ interior design, $14.8 \%$ landscape design, $12.9 \%$ new residential, $11.5 \%$ commercial, and $1.5 \%$ institutional projects. $32.2 \%$ of projects were won by designers from the United States, 42.4\% from Europe, and the rest was shared by designers from Asia, Africa and Australia ${ }^{6}$ (figure 1).

\section{COLLABORATION VS COMPETITION}

In traditional competitions teams or individuals beat one another. It is a competitive process based on prescribed rules and regulations that facilitate a fair race. Even in the best-case scenario, however, there is only one clear winner and the remainder are by definition on the losing end. In other words, all but one winner, experiences some sense of jealousy, disbelief or disappointment. Is there a possibility to have multiple winners? Or, what would happen if all designers could use any designs of any other entrant for their own iterations? What are the required intellectual protections?

\section{Exchange Model}

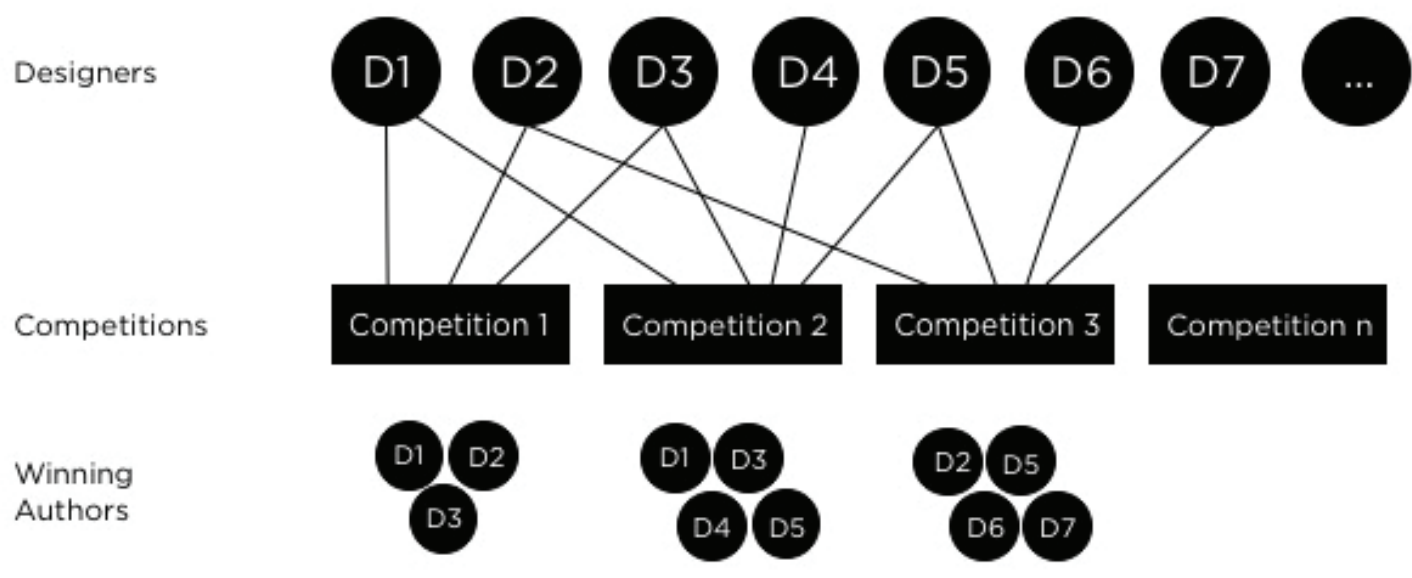

\section{Iterative Model}

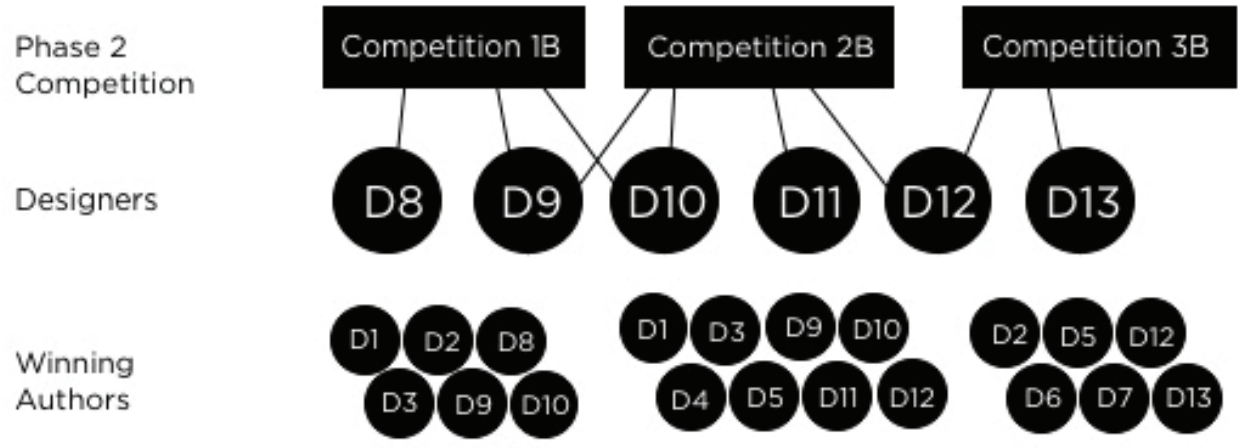


Designers often build teams to participate in competitions. Every team member brings ideally something complementary to the table. The team works together, shares the burden, and eventually benefits or suffers equally from the success or demise. However, is it possible to turn the competition model into a collaborative one, where participants do not compete but collaborate on projects? At Arcbazar, we explored the use of two collaborative models, a. the exchange model, and b. the iterative model (figure 2):

a. Exchange model: This method of team building happens through direct complementary contributions, i.e. say designer A produces a base drawing, designer $B$ uses the base drawing and takes designer A for an agreed upon equity into the team. Complimentary work can range from drawings, to environmental consulting, engineering input, partial design solutions, or any other project-related help. Designer A, in this way, can become part of multiple teams that agree on the terms put forth. Designers who do not have much time at their hands, or lack expertise in certain areas can still add a "brick on the wall," and potentially become part of a winning team. It fundamentally lowers the barriers of entry for designers to participate in competitions.

b. Iterative model: This method is an iterative process through a two-staged crowdsourcing protocol. In the first phase all designers submit their designs, the projects are evaluated and ranked. In the second phase, all designs are open for partial re-use by all other designers. The knowledge produced in the first stage is not lost but developed further.

In science, for comparison, an article often has multiple authors, and the order of authors reveal the degree of each individual's contribution to the work. In a similar way, entries in crowdsourcing projects can be "authored" by multiple designers based on their contributions. When, and if, the entry wins a competition, the award is shared according to the set equity distribution. If the design gets built, a team of designers gets credited as its' authors.

The collaborative models put forth here aim to facilitate a fair protocol and crediting mechanism for designers. They build upon aggregate design data by harvesting collective design intelligence.

\section{EVALUATION PROTOCOL}

One of the controversial parts of competitions are the evaluations of design entries. According to Moussavi the evaluation process in competitions has less to do with the merits than with the "theatre of unpredictability within which competitions unfold." ${ }^{\prime 7}$ There is no particular framework that could be applied uniformly and objectively on each project. Therefore, evaluations in traditional competitions often follow the tournament model, i.e. designs get judged comparatively and eliminated one by one until a clear winner emerges. Or, projects are judged on an additive basis, i.e. adding virtues, experimental quality and innovation, and the one with the most aggregated value gets selected. In either case, there are a limited number of possible outcomes for entries: a. the design wins the competition, $b$. the design loses it, c. the design gets built through repurposing it for another project, or $\mathrm{d}$. the design becomes part of a new solution by another architect. All but one project will end up in the latter three categories.

In crowdsourcing projects, on the other hand, evaluations are based on quantitative and qualitative design criteria. Quantitative criteria involve voting procedures among designers, family and friends of the project-owner, as well as scores on sustainability and budgeting. Altogether they provide a total score, which the system uses to rank each project. This type of automation in evaluation is essential, since the number of entries can often get overwhelming. There is an illustrative example from 1983, which shows such a problem in traditional competitions: The competition for the Paris Opera House received 756 entries that were pinned-up on a three-kilometer-long stretch of land.$^{8}$ It is literally impossible to have a fair evaluation through traditional reviews with that number of entries, no matter how well the jury, regulations, intentions and organization might be. Quasi-automated evaluation mechanisms as above-mentioned can offer a solution for this type of problem. Qualitative criteria, on the other hand, are derived by the client's own critique, written feedback from experts, and opinions from family and friends. In this setting, the project-owner is sanctioned to make an informed decision based on these data points. The evaluation process overall becomes transparent, and the competition outcome remains merit-based.

Tracking Designer Performance: Moreover, performance in online platforms does not always mean winning a contest. It can be more nuanced. For example, awards can be distributed in a more equitable way. Design entries can be evaluated, scored and ranked automatically, and the award can be distributed afterwards among all entrants based on relative scores. In this scenario, every qualified entry could get a share of the award. In other words, if there are, say, ten design submissions, each designer receives a proportional percentage of the total award - based on their final scores. In addition to monetary rewards, designers can also build up histories, collect points for various acts, i.e. signing up for a competition, submitting their entry, making peer evaluations, sharing data with peers, consulting project-owners, and so on. At Arcbazar, these points define the ranking of designers on performance charts. These can be filtered by location or type of design, i.e. charts of top European designers, landscape designers, etc. (figure 3). A designer can thus perform not only by winning a competition, but by contributing to the larger community. In fact, it has been argued that "peer consumption and feedback are important motivators of participation in crowdsourcing operations and online communities in general."9 


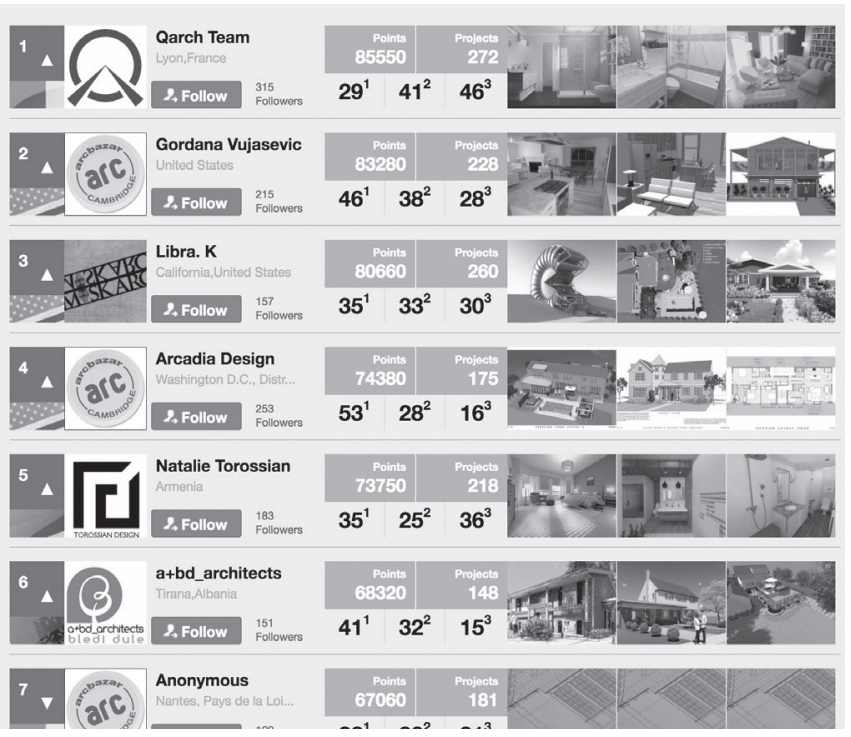

Figure 3. Screenshot of designer ranking charts on Arcbazar.

\section{ARTIFICIAL INTELLIGENCE IN DESIGN}

In the late 19th century, the city of Quebec organized a competition for the city hall. There were six design entries from Canada and the US, none of which satisfied the jury. The city decided to produce a composite design made from bits and pieces of all competition entries. The final design by Georges-Émile Tanguay became a "Frankenstein" composition, incorporating Romanesque, Neo-classical, and Neo-Gothic style features. Such a process was quite common in historic competitions. Today, stitching together a new design from various competition entries is certainly not considered acceptable or ethical. However, one could argue that decomposing projects and recombining the best aspects of each design entry into a new composition may offer the most ideal solution for a given design problem.

In 2017, we collaborated with a group of computer scientists from Raytheon through a DARPA (Defense Advanced Research Projects Agency) grant, to work on artificial intelligence in design. The goal of the research was to train deep neural networks with design data derived from Arcbazar to compose new conceptual designs - by piecing together high performing building blocks from completed designs in the database ${ }^{10}$ (figure 4).

Deep learning systems are powerful to analyze and discriminate information and identify latent patterns in a given dataset. However, their output is data-driven, i.e. historybased, and cannot produce anything entirely original. For example, when using a database of sports cars, the system cannot generate compositions of SUVs. Therefore, we worked on a new form of neural networks, called generative adversarial networks (or GANs), which are able to produce original variations not seen in a given dataset.
The long-term vision of this research is to develop an Al engine that can automate conceptual designs. For example, a project-owner defines a building program and provides the location of the project. The Al engine then infers climate data, lot boundaries, local zoning regulations, building codes, and any other relevant information from the location, and generates a series of design options. If wanted, these designs can be wrapped with particular vocabularies, e.g. classical, modernist, or styles of renowned architects, and tailored to a unique building program. The project-owner can then pick one of the designs and hire a local designer for implementation. Eventually, Al design bots may even participate in real-world crowdsourcing projects and become incorporeal competitors to corporeal designers (figure 5).

Architects are increasingly relying on digital extensions -CAD software, cloud services or ambient design knowledge provided by smart apps, which capture and process surroundings, draft designs, assist with design direction, and eventually may even design for us. Sooner or later we will face the existential question: what is our role as architects? Anything that can be quantified somehow will be eventually performed better, faster, and more efficiently through automation. In the short term, designers will benefit from Al-driven CAD tools and generate a multitude of conceptual designs to jumpstart their design process. In the long run, however, there might be consumer tools that are used directly by clients to generate and implement various solutions for their design problems. This will inevitably have significant bearings on the architectural practice, and new practice models might emerge. "This is wild speculation, but perhaps architectural practice could follow other creative fields, such as the music industry. For example, say, Frank Gehry develops a "style" and whoever uses his language through an Al-driven system pays a royalty. Gehry in that way might "design" millions of structures around the world..."11 This particular business model may or may never materialize, but the impact Al will have on architecture will help the penetration of high-end designs to even the most mundane design problems.

\section{CONCLUSION}

Crowdsourcing platforms are built on the traditional competition model but offer unique opportunities to expand high-end design to a global audience. Crowdsourcing overthrows, relocates, and drastically redefines our notions of design acquisition, clientele, and architectural practice. Any designer anywhere in the world can turn on their computers a workstation at their office, a desktop at home, or a laptop at a coffee shop - and log into a digital workspace. They can click on an icon and view running competitions, filled with a list of projects from around the world - a church in Myanmar, a retail store in Cambridge, an attic space in Cleveland, a villa in Rio, social housing in Nairobi, a floating entertainment island for lake Michigan in Chicago, and so on. The projects may be far away from the location of the designer, but with the 
help of new technologies the space-time continuum is not as important. Designers can view recorded video descriptions of the project, chat with project-owners over social media and video conferencing tools and collaborate with peers online.

This paper discussed the reincarnation of the traditional competition model through online crowdsourcing platforms taking the fair and open competition protocol to smaller scale projects around the globe. It elaborated on the development of new mechanisms to facilitate collective design generation, speculated on the impact of Al systems, and their potential effects on architectural practice. New media, emerging technologies, and novel forms of competitive models will inevitably push architects towards constant progress and innovation. Competitiveness is part of our human condition, and the hope for self-realization often drives great innovation and achievement.

\section{ENDNOTES}

1 Kyle Steinfeld, "Dreams May Come," in Acadia 2017 Disciplines \& Disruption: Proceedings of the 37th Annual Conference of the Association for Computer Aided Design in Architecture, eds., Takehiko Nagakura et al. (Cambridge, MA: The MIT Press, 2017), 590-599.

2 Helene Lipstadt, Experimental Tradition: Essays on Competitions in Architecture, (New York: Princeton Architectural Press, 1989), 10.

3 Jorg H. Gleiter, "Das Neue Ornament: Zur Genealogie des neuen Ornaments im Digitalen Zeitalter," Arch + 189 (2008): 76-83.

4 Lipstadt, Experimental Tradition, 15.

5 Adriana Bujor and Silvia Avasilcai, "Innovative Architectural Design Development: The Arcbazar Creative Crowdsourcing Contests Perspective," MATEC Web of Conferences 184, July 31, 2018.

6 Arcbazar official website statistics page, accessed September 23, 2018. https://www. arcbazar.com/map.

7 Farshid Moussavi, "Viewpoints: Farshid Moussavi on Competitions: 'Creative Leaps in the Arena of Architectural Competitions,'” The Architectural Review, January 31, 2013.

8 Hilde De Haan, Architects in Competition: International Architectural Competitions of the Last 200 Years (London: Thames and Hudson, 1988).

9 Elizabeth Keslacy, "Arcbazar and the Ethics of Crowdsourcing Architecture," Thresholds 46 (2018): 311.

10 Imdat As, Siddharth Pal, and Prithwish Basu, "Artificial Intelligence in Architecture: Generating Conceptual Design via Deep Learning," International Journal of Architectural Computing 16, no. 4 (2018)

11 Michael J. Crosbie, "Doom or Bloom: What Will Artificial Intelligence Mean for Architecture," Common Edge, September 17, 2018.

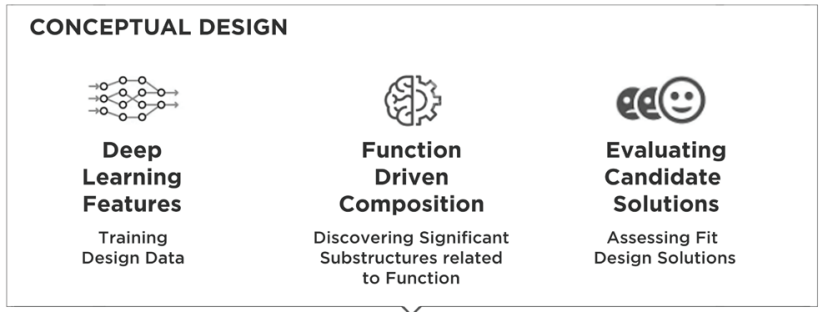

CANDIDATE DESIGN

\begin{tabular}{|ll|}
\hline PRELIMINARY DESIGN & > TOPOLOGY OPTIMIZATION \\
\hline DETAILED DESIGN & > PROXY OF PHYSICAL SYSTEM \\
\hline
\end{tabular}

Figure 4. Deep Learning system, training neural networks with design data from Arcbazar, generating function-driven design compositions, and evaluating the resulting solutions. The preliminary design goes through topology optimization and produces a graphic proxy of the final design.

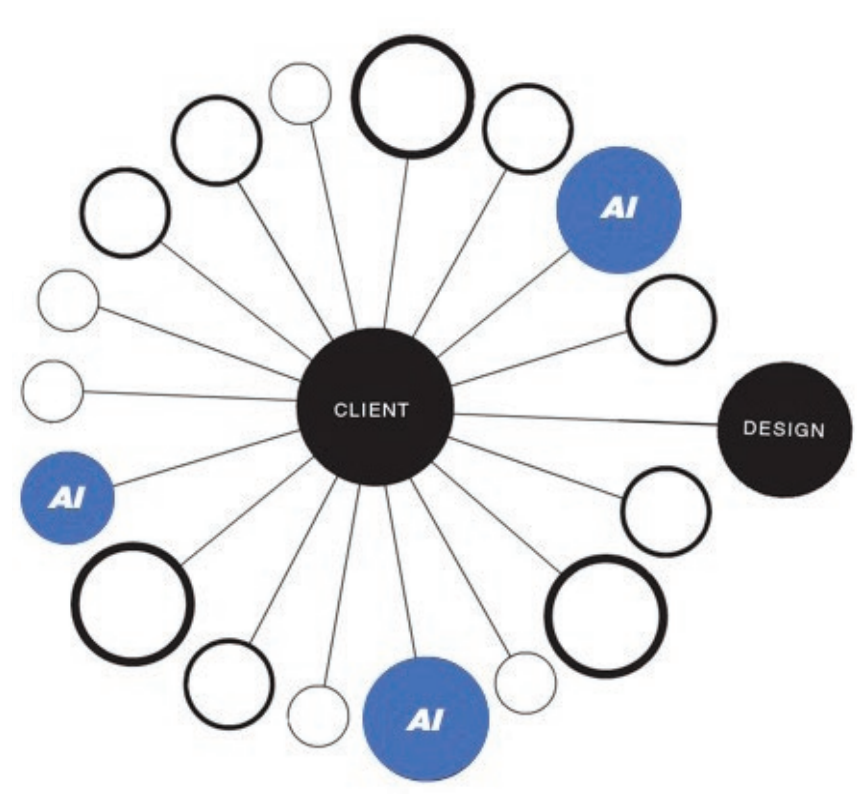

Figure 5. Hybrid competition model. Al bots and human participants competing in a crowdsourcing project. 